\title{
POSSIBILITIES OF THE JOINT USE OF OPTICAL AND RADAR DATA IN FLOOD SPACE MONITORING
}

\author{
O.P. Arkhipkin*, G.N. Sagatdinova \\ National Center of Space Research and Technology, 15 Shevchenko Street, Almaty, Kazakhstan - (oarkhipkin, \\ gulshatn)@rambler.ru
}

ISPRS Commission III, ICWGIII/IVa

KEY WORDS: Remote Sensing, Space Monitoring, High Waters, Flood, Radar Data, Radar Polarimetry

\begin{abstract}
:
The article gives a brief description of the system of space monitoring of high water and floods. Its main tasks are the operational dynamics of snow and ice cover melting and the passage of flood waters. The solution of these tasks is carried out in three levels corresponding to the low, medium and high resolution of remote sensing data. An important role in monitoring is given to radar data. This is due to the features of the radar survey: independence from weather conditions and time of day, regularity, good spatial resolution, the possibility of using polarimetric properties (including phase information). The use of radar data also provides additional information, including the allocation of wet soils, flooded vegetation and infrastructure. The presence of large time periods of repeated survey, interference (cloudiness, haze, noise, etc.), different spatial resolution necessitates a complex analysis of optical and radar data in flood space monitoring. Such analysis makes it possible to better observe the flood dynamics, more precisely identify of flooding zones and determine their structure. Features of radar survey (transparency of dry snow and change of reflected signal during snowmelt) allow using them to determine the beginning of snow melt and determine the degree of water content in it. Optical data are also used to determine the area and structure of the snow cover. Method of detecting the beginning of the snowmelt period consists in the comparison of the current radar image with a base image created as an average image from the winter images with dry snow.
\end{abstract}

\section{INTRODUCTION}

For more than 15 years our organization has been engaged in the development and implementation of emergency space monitoring technologies in the practical activities of Kazakhstan emergency authorities at various levels. When we started these works in 2001, space monitoring of emergencies was not used in the work of emergency authorities. Over the following years, we implemented operational space monitoring of fires in all regions and space monitoring of high water and flood in eight regions of Kazakhstan.

The system of space monitoring of high water and flood is a constantly developing system. Development is carried out by introducing new tasks, methods, technologies, new Remote sensing satellite systems and etc. into the system. In the development of the system various methods and technologies of processing optical and radar remote sensing data were introduced (Arkhipkin and Sagatdinova, 2008), (Arkhipkin et al., 2007), (Arkhipkin et al., 2010) and (Spivak et al., 2004). At the current time, the system is a three-level structure whose levels are determined by the spatial resolution of the used Remote sensing data. Initially, only the first level (low resolution) data was mainly used with irregular use of the second and third level data. The situation has significantly changed in the last few years due to the appearance in the open access of medium and high resolution data. In 2014-2015 the optical Landsat- 8 and radar Sentinel-1A medium resolution data and in 2016 optical high resolution data from the Sentinel-2A satellite (spatial resolution $10 \mathrm{~m}$ ) have been entered into regular use.

\footnotetext{
${ }^{*}$ Corresponding author
}

In recent years considerable attention has been focused to methods and technologies of complex analysis of optical and radar data of different spatial resolution and radar polarimetric methods (Arkhipkin and Sagatdinova, 2016) and (Arkhipkin and Sagatdinova, 2017). A joint analysis of optical and radar data of different resolution allows, on the one hand, to better identify flood zones and determine their structure, and on the other, it is better to monitor the dynamics of high water and flood. The need for such a comprehensive analysis is due to several reasons: different spatial resolution, different time period of repeated survey, interference during survey (cloudiness, haze, noise interference, etc.). The use of radar polarimetry methods allows obtaining a new various of additional information in comparison with one-polarizing data. In particular, they allow identifying moist soils and flooded vegetation and infrastructure.

The main objectives of space monitoring of high water and flood are to determine the current state of the flood situation and to assess the dynamics of its development both with the help of a time series of current satellite imagery and with the use of modelling methods. A feature of the current stage of the development of the system of space monitoring of high water and flood is the research of the period preceding the beginning of the passage of flood waters in order to forecast the time period of the beginning of the flood and forecast using radar methods and methods for modelling the initial stage of its development. 


\section{A BRIEF DESCRIPTION OF SPACE MONITORING SYSTEM OF HIGH WATER AND FLOOD IN KAZAKHSTAN}

\subsection{General features of the system}

Functionally, the system of space monitoring of high water and flood can be divided into several blocks. The most important of these is the block of operative space monitoring. Also data transfer block is regularly used to create on the results of monitoring output data and transmit them to users. If necessary the survey and analytical blocks and also the modelling block can be used additionally. The main task of the survey block is to summarize the results of monitoring in a particular territory for a certain period of time (week, decade, month, season, etc.). The analytical block is designed to determine the dynamics of high water and flood development on the basis of analysis of seasonal and long-term time series of space monitoring data on flooding zones. The block of modelling allows assessing the potential course of flood development in the GIS on the basis of the current flood situation and the forecast of meteorological data. The current flood situation is estimated from the space monitoring data, and the forecasted meteorological data is taken via the Internet from global forecast meteorological systems (for example, GFS). At the current period this block is in a stage of trial operation.

When carrying out space monitoring of high water and floods its main tasks are:

- Operative space monitoring of a destruction of the snow and ice cover;

- Operative space monitoring of the passage of high water and flood.

At the beginning of the monitoring only the first task is solved. Later, when melt-water appears, both tasks are done together, and after the descent of snow cover - only the second task. Space monitoring of the dynamics of the filling of the most important reservoirs, as well as a number of other important water objects, is singled out as a separate task. Here the dynamics of the destruction of the ice cover is also done.

As already noted above our system of space monitoring of high water and flood has a three-level structure, respectively Remote sensing data of low, medium, high and ultra-high resolution. The solution of the above tasks at the first level is based on receiving stations in Almaty and Astana. They provide several times a day reception of data from the US satellites EOS (Terra and Aqua) and Suomi NPP on-line. These data are obtained daily and cover the entire territory of Kazakhstan. This allows us to operative assess the current state of the flood situation and monitor the dynamics of its development. As for reservoirs, at this level the surface of the water only of large and big reservoirs is defined.

At the second level the specification of the flood situation for separate territories are detailed on the basis of medium resolution data. As a base at this level, Landsat- 8 optical data and radar Sentinel-1 A and Sentinel-1 B data are used. These data are regularly displayed in free access. Despite the fact that these data cover a not very large area and have a period of recurrence of 12-16 days, they are very useful and informative for monitoring the flood situation. Once again, we emphasize the importance of using radar data in the flood monitoring. This is caused, on the one hand, to the fact that they are not affected by the time of day and any cloudiness. On the other hand, the features of the Sentinel-1 data structure (dual polarization and phase information) also allow additional studies of the underlying surface.

Even more detailed information is formed at the third level for small areas based on high resolution and ultrahigh resolution satellite images, the use of which usually requires significant financial resources. In critical cases for these purposes it is possible to obtain data from relevant international organizations (for example International Charter Space and Major Disasters) that supply them free of charge. Great hopes at this level are assigned to the use of such data from Kazakhstan's KazSat 1 and 2 satellites, which already operate in the industrial operation mode

\subsection{Output information of operative space monitoring of high water and flood}

In the process of operational space monitoring in the winterspring period, the following map are formed:

- Maps of snow and ice cover and high water passage with the allocation of flooding zones based on low resolution Terra and Aqua MODIS data (daily).

- Maps of snow and ice cover and high water passage with the allocation of flooding zones based on medium resolution Landsat and Sentinel (as they are received).

The main task of space monitoring of a descent of snow cover and the passage of flood waters is the allocation of flooding zones. For its decision on each level is carried out division data to water and non-water objects. In each of these main classes it is possible to allocate additional classes of the underlying surface depending on the task and level. So, on water objects are allocated on water and ice covered surfaces, and on nonwater objects are allocated on snow and snow-free areas. From the class of snow-covered areas we distinguish zones of active snowmelt (areas where the surface temperature is more than $0^{\circ}$ C).

At any level all water objects can be divided into constants (stable) and zones of flooding which are defined as a difference of the current surface of the water and a constant. Mask of constant water objects are formed on basis of Landsat images in non-flooded season, mainly by autumn images. For data of high and ultrahigh resolution the archival pictures or data downloaded from the Internet satellite maps are used for these purposes.

For optical imagery of any level we must introduce an additional class areas covered by a cloud cover, which does not allow us to determine the state of the underlying surface.

On the results of the thematic analysis are constructed two types of operative maps: regional and district. The regional maps reflect the situation in the whole region. On district level flood situation is presented in more large scale with additional GIS layers (settlements, roads, pipelines, power grids, etc.) according to the user requests.

Figure 1 presents a sample of the output information of operative space monitoring of high water and flood at the regional level, obtained from low resolution MODIS data. This map is formed in the period when the snow cover still exists and the high waters have already started flowing. On it the cartographic information is minimized, so as not to obscure the overall picture with the flood situation. 


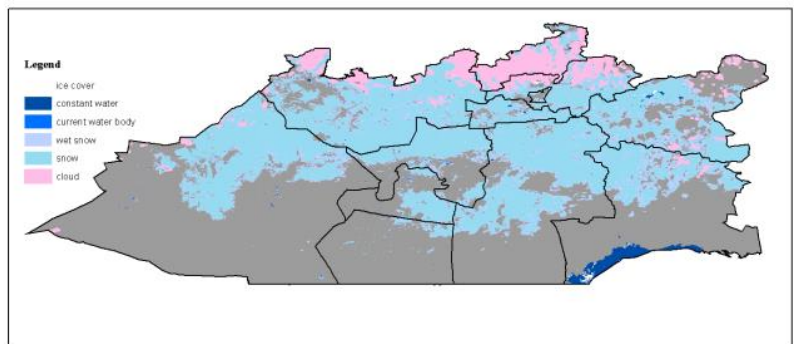

Figure 1. A sample of the operative output information of space monitoring of a descent of snow cover and passing of high waters according to MODIS at the regional level

A sample of the output information of the operative space monitoring of high water and flood at the district level, obtained on the basis of low-resolution MODIS data, is shown in Figure 2. This map formed in active phase of the high water. At the district level cartographical information is provided more widely in comparison with regional level. The content of cartographic information depends on user's wishes, but almost always there is a cartographic layer containing the location and name of settlements. This information is necessary both for better orientation in spatial location and for assessing the potential threat of floods. Occasionally users asked to use space images or topographic maps or relief as a substrate, and also to overlay a grid on maps.

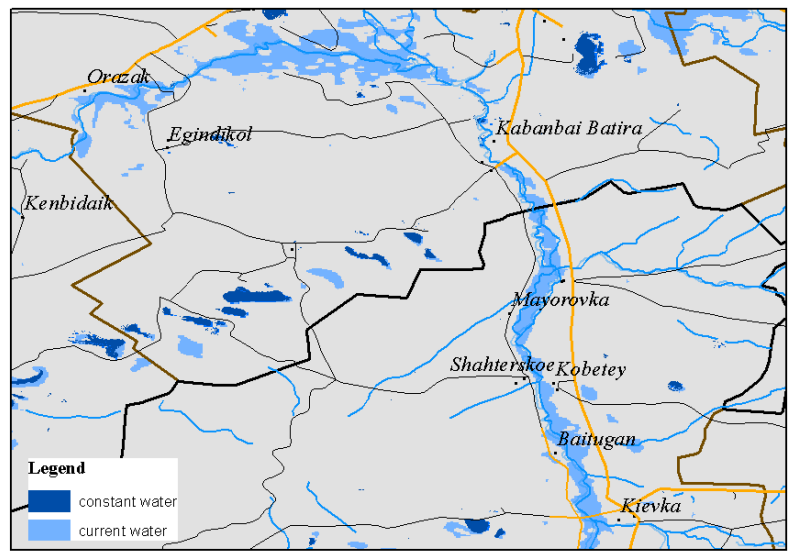

Figure 2. A sample of the operative output information of the space monitoring of the passage of flood waters according to the MODIS data at the district level

The output data obtained from the MODIS data is send to users about an hour after the reception of the space images. Medium resolution data require much more time for processing, but they also send users with greatest possible operativeness. As an example Figure 3 shows such data for a fragment of the Irtysh river basin, located in the Pavlodar region.

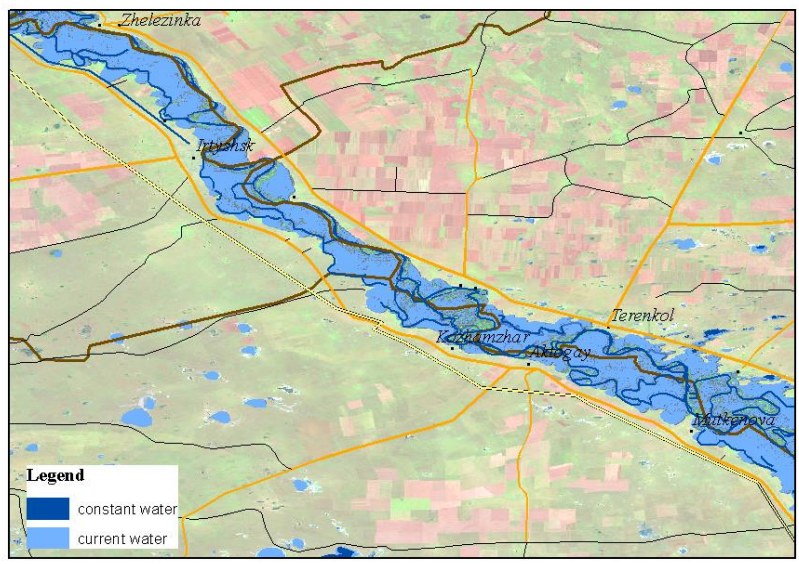

Figure 3. A sample of the operative output information of the flood space monitoring according to Landsat- 8 data

The transfer of the output data of the space monitoring of high water and flood to regional and district emergency managements is carried out by means of e-mail. We have also developed more modern technologies for data transferring through a geoportal or with the use of situation centers of emergency, but they have not been used to the right extent.

\section{DYNAMICS OF FLOODING ZONES OF ON THE BASIS OF JOINT ANALYSIS OF OPTICAL AND RADAR DATA OF DIFFERENT RESOLUTION}

The results of operative space monitoring of high water and flood allow assessing the current flood situation. These results form a consistent time series, the analysis of which allows us to assess the dynamics of the development of the flood situation. Such series are formed for each of the used optical and radar remote sensing data, which also differ in spatial resolution. Every type of this series has significant omissions. In optical data, these omissions are due to cloudiness; for medium resolution data they are caused by a long period of repeated survey. A joint research of all these series allows us to obtain a more complete and qualitative picture of the dynamics of the development of the flood situation.

Radar data plays a particularly important role in flood monitoring, since often optical data has large omissions due to cloudiness in the most active phase of the development of the flood situation. For example, long-term observations in Kazakhstan have shown that in the peak flood period from 50 to $90 \%$ of the MODIS data cannot be used to monitor floods due to high cloud cover. Receiving radar data in the presence of high cloudiness allows filling gaps in information about the development of the flood situation and using them for joint analysis with optical data of medium or high resolution.

In connection with the above, it is desirable to increase the number of radar data used for flood monitoring. From this point of view, big expectations were associated with the introduction of the Sentinel-1 B radar satellite into industrial operation in 2017. Theoretically, this could double the number of radar data obtained in free access and halve the re-survey period. However the practice has shown other result. For the regions we observed, instead of Sentinel-1 A radar data Sentinel-1 B data was provided, and Sentinel-1 A data was not provided at all. 
The use of other systems of radar data on a paid basis was hampered by significant deficiencies in their order. The main weaknesses of the widespread use of radar data in the operative monitoring of high water and flood are their high cost and difficulties of operative order. Earlier it was required to determine previously the location and the time of survey at $100 \%$ advance payment, which did not allow to take into account the rapidly changing situation during the passage of high water and flood. At last time the situation with the operative order is changing. For example Airbus Defense and Space has put in place a special procedure that allows you to quickly determine the location and time of survey based on the current situation, and also to organize an operational survey and the provision of radar images (Besnard, 2015). This procedure was tested by us in the operative monitoring of high water in the third decade of April 2015.

The selection of scenes for the operative ordering of high resolution radar data TerraSar-X (StripMap mode, spatial resolution $3 \mathrm{~m}$ ) was carried out on the basis of an analysis of the dynamics of the development of the flood situation using lowand medium-resolution optical data. According to its results, zones of active high water were identified. For these zones, the potential scenes for survey for the 1-3 days were considered. In a choice of scenes and survey dates was estimated not only the area but also trend of changes. As a result of the analysis, 5 scenes were selected for the operative radar survey of zones with the most severe flood.

In addition to the possibility of operative radar survey critical importance has a time of receiving, processing and sending output data to emergency centers. Time of delivery data after survey TerraSar-X data on FTP consists less an hour. Downloading and processing received data constitutes several hours. In our opinion, this is a good indicator of efficiency for high resolution data. It should be noted that this data was obtained in a period of high cloudiness, what allowed controlling flood situation at these days.

The use of medium and high resolution data provides a more detailed picture of flooding zones. The joint use of such optical and radar data makes it possible to form a more representative unified single time series of medium and high resolution data. In conclusion of this section, we will give an example of the integrated use of the TerraSar-X and Sentinel-1 A radar data and optical Landsat- 8 in the central part of the Akmola region. Figure 3 shows the dynamics of flood development between April 19 and 22, 2015, obtained from radar data of different resolution (TerraSar-X and Sentinel-1 A), and Figure 4 shows the dynamics of flood development between April 22 and 30, 2015 year, obtained by radar (TerraSar-X) and optical (Landsat8) data.

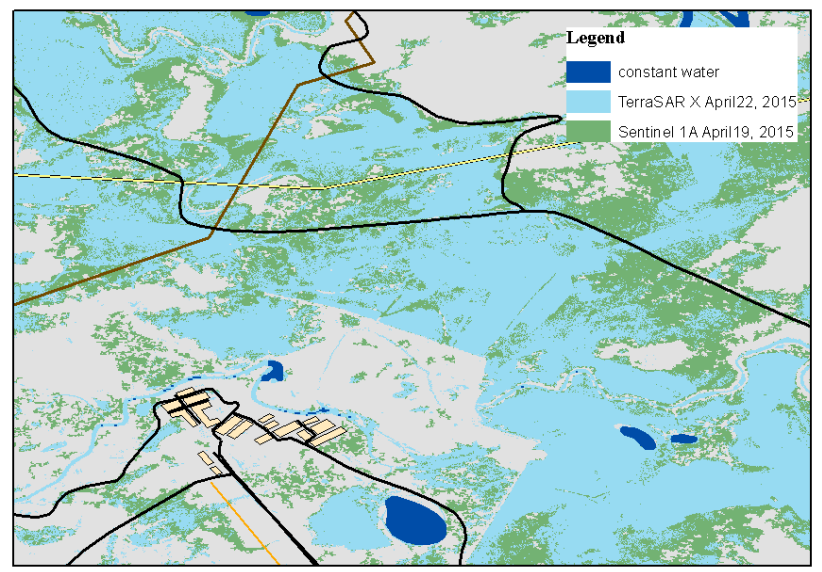

Figure 4. Flood dynamic in the period between 19 and 22 April 2015, received by TerraSar-X and Sentinel-1A data

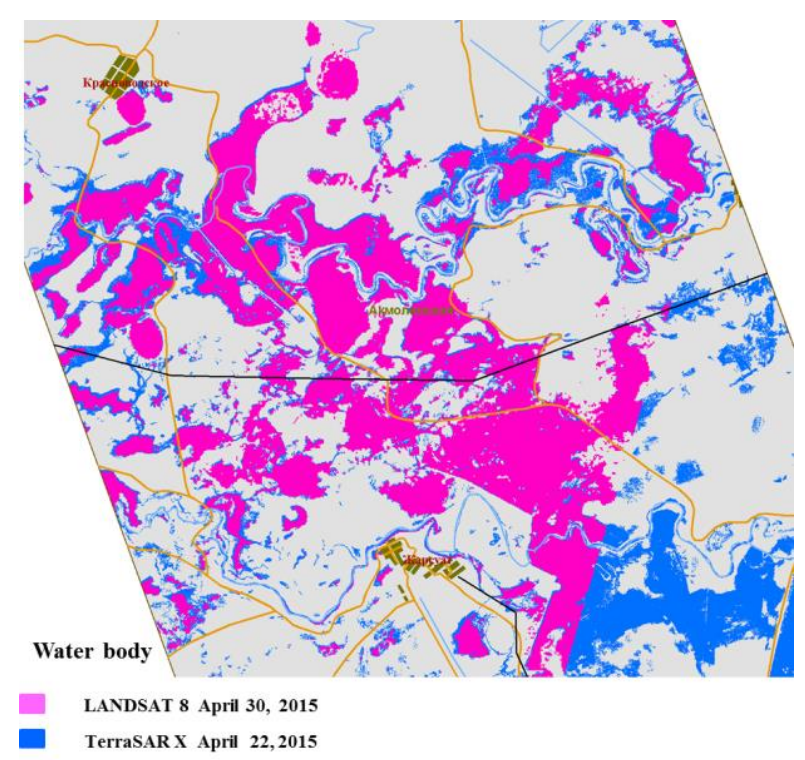

Figure 5. Flood dynamic in the period between 22 and 30 April 2015 , received by TerraSar- $X$ and Landsat -8 data

\section{THE USE OF RADAR AND OPTICAL DATA FOR THE FORECAST OF THE INITIAL PERIOD OF SNOW MELT AND A POTENTIAL DANGER OF FLOOD}

\subsection{Statement of the problem of forecasting the occurrence and development of high water}

Until recently, the main task of space monitoring of high water and flood was to determine the current state of the flood situation and to assess the dynamics of its development both with the help of a time series of current satellite imagery and with the use of modeling methods. A special feature of the task presented here is the research of the period preceding the beginning of the passage of flood waters. The purpose of such researches is studying of a possibility of the forecast of time of the flood beginning. 
The importance and actuality of this task was shown by events related to the passage of flood waters in the spring of this year. In this year a large high water was expected in many regions of Kazakhstan and the authorities were preparing for it. However, its beginning for some regions was unexpected both in time and in scale, which led to an increase in material damage.

The accuracy of assessments of current methods of forecasting the potential passage of high water is still insufficient, especially in the initial period. Ground-based methods using Remote sensing data as auxiliary have significant drawbacks, which are as follows (Kuchment, 2010). Physical models of the snow cover suggest the solution of balance equations in the cells of a regular grid. The solution of balance equations was theoretically well developed in the last century. However, it requires a large number of initial data, which usually ground methods can not provide (Pyankov et al., 2016). This is due to their significant shortcomings, consisting in a small number of weather stations and snow surveys, which do not allow to take into account for the heterogeneity of the snow field due to the influence of the relief and the diversity of the vegetation cover. But also it is difficult to make representative routes for snow measurements for territories with a complex terrain. During the spring snowmelt, the representativeness and reliability of the snow survey data is further reduced, and the frequency of their obtaining becomes very inadequate.

Along with ground methods, methods for estimating the amount of accumulated snow moisture on the basis of Remote Sensing data, which were mainly developed for the microwave range, also developed (Chang, Rango, 2000) and (Macelloni et al., 2001). For this range there is a relative transparency of the atmosphere. Radiation depends on the state of the underlying surface, including the presence of snow cover and its depth. The attenuation of the intensity of this radiation after passing through the snow cover is proportional to its moisture reserve, which is the basis for the development of such methods. These methods are developed over several decades, but the accuracy of their estimates is still insufficient. Therefore, their use is very difficult, especially during the active snowmelt. There are several reasons. The main one is that wet snow has the same microwave properties as the snow-free surface. This makes it impossible to determinate snow parameters in snowmelt period or thaw. In addition, the signal is affected by the presence of forest cover, graininess of snow, changes in snow structure and a number of other factors. Attempts are being made to take into account the influence of these factors, but the problem has not been completely solved yet. The accuracy of the methodology does not allow using it yet.

Complex methods for estimating snow cover moisture reserves are carried out on the basis of a combination of ground and aerospace observations. Among these systems, we highlight the The Global Data Assimilation System (GDAS). This system is formed under the aegis of NOAA and generates information on a worldwide scale. GDAS is a comprehensive data analysis system that includes data from ground, balloon, aircraft, space, ground-based radar and buoyant observations. Based on the results of the complex analysis, every six hours with a spatial resolution of 0.25 degrees large sets of output data of various meteorological parameters are formed. For tasks of analysis and prediction of such hydrological phenomena as flood, particular interest is provided by data on the depth of the snow cover and the water equivalent of the snow cover. Data from current global meteorological parameters obtained in global systems are used to predict the dynamics of their changes using various software model complexes, including the Global Forecast System (GFS) and the Weather Research and Forecasting (WRF) Model. They are widely used in modeling the processes of snow accumulation and snow melting (Zhao et al., 2009).

In recent decades, the direction to use the data of radar space surveys for the analysis of snow cover characteristics has been actively developing (Pivot, 2012), (Koskinen et al., 2010) and (Yamaguchi et al., 2014). At the same time possibilities of use for these purposes of radar data of all three ranges applied in Remote sensing (X - 3,1 cm, C - 5,6 cm and L - 23,5 cm) for all four polarizations were researched. The application of a radar survey to assess the state of the snow cover has its own characteristics, which essentially distinguish them from passive microwave survey. Firstly, dry snow cover is almost transparent for radar survey and isn't fixed by it directly. Secondly, the appearance of water in the snow cover is reflected in the radar image and this makes it possible to use radar data to fix the wet snow cover

4.2 Methodology of using radar and optical data to determine the beginning of snow melt and an estimate of the water content in it

Features of radar imaging in the C-band allow the use of Sentinel-1 data to determine the beginning of snow melt and estimate the degree of water content in it. To detection wet snow by radar data, a special technique has been developed that takes into account the specific features of the radar survey of the snow cover. Such a selection is not carried out directly by the radar image. It is carried out by comparison with the base image, which is the average value of the backscatter factor in each pixel for all pictures with a dry snow cover. The basic image is introduced in order to level out the influence of variations of backscattering value of the underlying surface, caused by variations in the survey conditions. It is formed in each pixel by the formula

$$
\sigma_{r e f}=\frac{\sum_{i=1}^{\mathrm{n}} \sigma_{\mathrm{n}}}{n}
$$

where $\sigma_{\text {ref }}$ - the value of the backscatter coefficient of the base image

$\mathrm{n}$ - the number of radar images obtained during the period of stable dry snow cover

$\sigma_{n}$ - the current value of the backscattering coefficient in these images

Comparison of the current value of the backscattering coefficient in the snow melting period with the base one can be carried out in several ways. In one of them, the difference (D) of the current $\left(\sigma_{w s}\right)$ and the base value is calculated in each pixel

$$
D=\sigma_{W S}-\sigma_{\text {ref }}
$$


In another way ratio $(\mathrm{R})$ of this two values is analyzed

$$
R=\frac{\sigma_{\mathrm{WQ}}}{\sigma_{\text {ras" }}}
$$

The values $\mathrm{D}$ or $\mathrm{R}$ can be calculated for any of the polarizations, and based on the results of the calculation the spatial distribution of wet snow and its state estimation are carried out. In each particular case, one can choose the best polarization for this. Methods are also being developed for the use of several polarizations at once to obtain snow cover characteristics.

There are other methods for estimating the snow cover characteristics based on radar data, including the height of the snow cover and the water equivalent. For example, for these purposes use such parameters of polarimetric radar data as entropy and angle $\alpha$.

Another important component of the technology is optical space images of different resolution, which allow you to track the actual situation with the descent of the snow cover and the passage of flood waters. First of all, they are used to determine the boundaries of the snow cover, since outside its boundaries the parameters $\mathrm{D}$ or $\mathrm{R}$ behave differently than within the boundaries. GDAS data are also used to estimate snow cover boundaries.

\subsection{Approbation of methodology of the forecast of appearance and potential danger of flood}

As the basic area for approbation of methodology the Nura river basin in the Karaganda region has been chosen. At the choice of the territory of a research the following criteria have been taken: - The existence of a representative number of radar data for these territories;

- The area has to support river network with not the really big, but not small pool located completely in the territory of Kazakhstan.

For this basin, the territory from which the melt water collected is determined. In the geographic information system QGIS, with help on HydroSHEDS BASINS data, a map of the sub-basins forming the Nura River basin was constructed. Every sub-basin include own part of surface water catchments area. For each sub-basin, maps of directions of surface water runoffs, as well as maps of water bodies, including rivers, their tributaries, lakes and reservoirs, have been constructed.

Also during the winter-spring period for it a database of archival and operative optical data was formed in 2017. These data included low resolution Terra and Aqua MODIS data and Landsat- 8 medium resolution data. Optical data are necessary for determining areas covered with snow. The database of archival and operative meteorological data was formed on the basis of the global GDAS data assimilation system. It contained data on the surface temperature, depth and water equivalent of the snow cover. These data were used for additional monitoring of snow-covered areas, as well as for assessing their potential flood hazard.
Approbation of the methodology was carried out in three stages. At the first stage, a base image was created, which is formed by calculating the arithmetic mean value in each pixel for all radar images of Sentinel-1 B during the period of stable dry snow cover (formula 1). In the second stage, parameters characterizing the distribution and the state of the wet snow cover (formulas 2-3) for each polarization were investigated on the basis of the current and base images. We also investigated complex parameters, which are calculated using several polarizations. An analysis of the results obtained showed that the best results are obtained for the ratio $\mathrm{R}$ of polarization $\mathrm{VV}$. Therefore, further research was carried out based on this parameter that was calculated in each pixel of the available radar data.

Parameter Rvv calculation in case of dry snow cover gives us dark grey surface, in case of water appearance in snow the surface becomes lighter. And the more water in the snow, the lighter the surface. In places where the water surface is formed, the image becomes dark. After the descent of the snow cover, the application of the parameter already gives nothing. Therefore, the specification of the snow cover borders by optical data and meteorological data is important. Its allow decrease methodology mistakes.

In the third and last stage, maps of snow melting zones were formed on the basis of classification using threshold values that make it possible to identify snow melting zones, spatial distribution of wet snow and estimate the degree of water content in the snow. The first appearance of these zones allows us to identify the initial period of snow melt. With the help of the value of $\mathrm{R}$, the dynamics of the melting of the snow cover is monitored. Figure 6 shows one of the options for presenting the final distribution map of wet snow. Here more dark color corresponds to the bigger content of water.

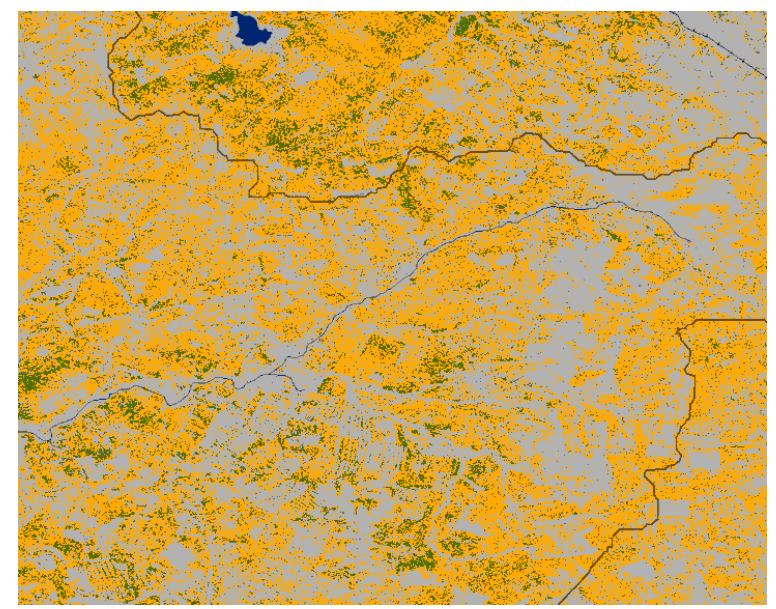

Figure 6. Mask of wet snow, obtained from the radar data of Sentinel-1B for April 4, 2017

Comparing the masks of distribution of wet snow in a few days, we obtain the dynamics of its distribution (Figure 7). 


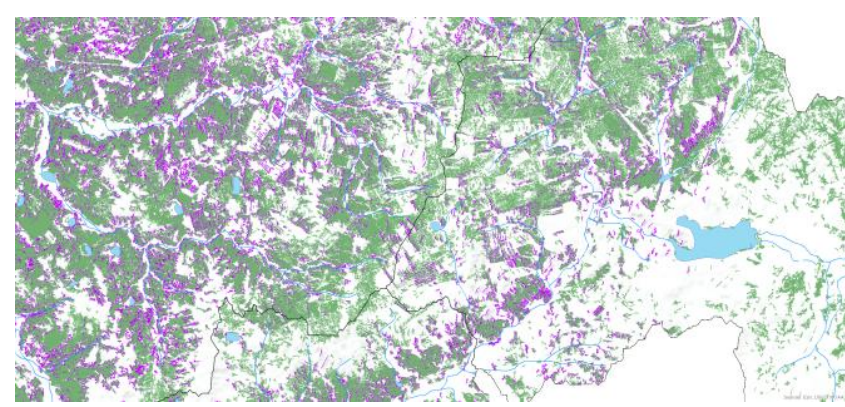

Figure 7. Dynamics of distribution of wet snow for April 4-9, 2017

To assess the potential threat of floods, the spatial distribution of the $\mathrm{R}$ value is compared with the spatial data of the GDAS on the water equivalent of the snow cover.

\section{CONCLUSIONS}

1. At present, space monitoring of high water and flood has become a working tool in the work of emergency authorities at all levels in Kazakhstan. However, so far his capabilities are used far not fully.

2. The introduction on a regular basis of the data of the medium resolution of radar Sentinel-1A and optical Landsat-8 allowed to significantly increasing the information content and the quality level of monitoring. Their joint use allows for a better and more detailed observation of the dynamics of flood development.

3. Estimating, in general, the use of radar data for monitoring snow cover in Kazakhstan, we can say that this direction is quite new and it is too early to make definite conclusions about the possibilities of its practical application. However, it is possible to use radar data to identify the beginning of the passage of flood waters, monitor the dynamics of flood development, and assess the potential threat.

\section{REFERENCES}

Arkhipkin, O.P., Sagatdinova, G.N., 2008. Functioning of Fires and Flood Space Monitoring System in Kazakhstan. In: The International Archives of the Photogrammetry, Remote Sensing and Spatial Information Sciences, Beijing, China, Vol. XXXVII. Part B8, pp. 435-439.

Arkhipkin, O.P., Sagatdinova, G.N., 2016. The use of various optical and radar remote sensing data in operative space monitoring of flood in Kazakhstan. Journal of Siberian Federal University. Engineering \& Technologies, 9(7), pp. 1045-1058.

Arkhipkin, O.P., Sagatdinova, G.N., 2017. The use of polarimetric radar data at space monitoring of high waters and floods. Sovremennye problemy distantsionnogo zondirovaniya Zemli iz kosmosa (Current problems in remote sensing of the Earth from space), 14(2), pp. 175-184

Arkhipkin, O.P., Spivak, L.F., Sagatdinova, G.N., 2007. Space monitoring of flood in Kazakhstan. In: Proceedings of the IGARSS'2007, Barselona, Spain, pp. 419-436.
Arkhipkin, O.P., Spivak, L.F., Sagatdinova, G.N., 2010. Geoscience and Remote Sensing New Achievements. In Teach, Croatia, pp. 419-436.

Besnard, M. (2015): Instant Tasking - Get Ready to Face the Unexpected. In: 3rd International GIS Forum "Integrated Geospatial Solutions - the Future of Information Technologies".

ftp://ftp.sovzond.ru/forum/2015/16.04_pdf/Besnard.pdf

Chang, A., Rango, A., 2000. Algorithm Theoretical Basis Document for the AMSR-E Snow Water Equivalent Algorithm, Version 3.1. Greenbelt, MD, USA: NASA Goddard Space Flight Center.

Koskinen, J.T.; Pulliainen, J.T.; Luojus, K.P.; Takala, M., 2010. Monitoring of snow-cover properties during the spring melting period in forested areas. IEEE Transactions on Geoscience and Remote Sensing, 48, pp.50-58.

Kuchment, L.S., Romanov, P.Yu., Gelfan, A.N., Demidov, V.N., 2010. Use of satellite-derived data for characterization of snow cover and simulation of snowmelt runoff through a distributed physically based model of runoff generation. Hydrology and Earth system science, 14(2), pp. 339-350.

Macelloni, G., Paloscia, S., Pampaloni, P., Tedesco, M., 2001. Microwave emission from dry snow: A comparison of experimental and model results. IEEE Trans. Geosci. and Rem. Sens., 39(12), pp. 2449-2656.

Pivot, Frédérique, 2012. C-Band SAR Imagery for Snow-Cover Monitoring at Treeline, Churchill, Manitoba, Canada. Remote Sens., 4, pp. 2133-2155.

Pyankov, S.V., Shikhov, A.N., 2016. Modeling the spatial distribution of snow cover on a large catchment area using satellite data. Sovremennye problemy distantsionnogo zondirovaniya Zemli iz kosmosa (Current problems in remote sensing of the Earth from space), 13(4), pp. 29-41.

Spivak, L., Arkhipkin, O., Pankratov, V., Vitkovskaya, I., Sagatdinova, G., 2004. Space monitoring of floods in Kazakhstan. Mathematics and Computers in Simulation, 67, pp. 365-370.

Yoshio Yamaguchi, Gulab Singh, Satoru Yamaguchi, Andrew C. Whitaker., 2014. Polarimetric SAR Response of SnowCovered Area Observed by Multi-Temporal ALOS PALSAR Fully Polarimetric Mode. IEEE Transactions on Geoscience and Remote Sensing, 52(1), pp. 329 - 340.

Zhao, Q., Liu, Z., Ye, B., Qin, Y., Wei, Z., Fang, S., 2009. A snowmelt runoff forecasting model coupling WRF and DHSVM. Hydrology and Earth Systems sciences, 13(10), pp. 1897-1906. 